\title{
The obscured X-ray source population in the HELLAS2XMM survey: the Spitzer view
}

\author{
Cristian Vignali*,†, Francesca Pozzi* ${ }^{*}$ Andrea Comastri ${ }^{\dagger}$, Lucia Pozzetti $^{\dagger}$, \\ Marco Mignoli ${ }^{\dagger}$, Carlotta Gruppioni ${ }^{\dagger}$, Giovanni Zamorani ${ }^{\dagger}$, Carlo Lari**, \\ Francesca Civano*, Marcella Brusa ${ }^{\ddagger}$, Fabrizio Fiore ${ }^{\S}$ and Roberto \\ Maiolino $^{\S}$ \\ *Dipartimento di Astronomia, Università di Bologna, Via Ranzani 1, I-40127 Bologna, Italy \\ ${ }^{\dagger} I N A F$ - Osservatorio Astronomico di Bologna, Via Ranzani 1, I-40127 Bologna, Italy \\ ${ }^{* *}$ INAF - Istituto di Radioastronomia (IRA), Via Gobetti 101, I-40129 Bologna, Italy \\ ${ }^{\ddagger}$ Max Planck Institut für Extraterrestrische Physik (MPE), Giessenbachstrasse 1, D-85748 \\ Garching bei München, Germany \\ $\S$ INAF - Osservatorio Astronomico di Roma, Via Frascati 33, I-00040 Monteporzio-Catone (RM), \\ Italy
}

\begin{abstract}
Recent X-ray surveys have provided a large number of high-luminosity, obscured Active Galactic Nuclei (AGN), the so-called Type 2 quasars. Despite the large amount of multi-wavelength supporting data, the main parameters related to the black holes harbored in such AGN are still poorly known. Here we present the preliminary results obtained for a sample of eight Type 2 quasars in the redshift range $\approx 0.9-2.1$ selected from the HELLAS2XMM survey, for which we used $K_{\mathrm{s}}$-band, Spitzer IRAC and MIPS data at $24 \mu \mathrm{m}$ to estimate bolometric corrections, black hole masses, and Eddington ratios.
\end{abstract}

Keywords: Galaxies: active - Galaxies: nuclei - X-rays: galaxies

PACS: 98.54.-h, 98.58.Jg

\section{INTRODUCTION}

Over the last six years, the X-ray surveys carried out by Chandra and XMM-Newton (e.g., [1, 2, 3]; see [4] for a review) have provided remarkable results in resolving a significant fraction of the cosmic X-ray background (XRB; [5, 6]), up to $\approx 80 \%$ in the 2-8 keV band (e.g., [7, 8]). Despite the idea that a large fraction of the accretion-driven energy density in the Universe resides in obscured X-ray sources has been widely supported and accepted (e.g., [9, 10]), until recently only limited information was available to properly characterize the broad-band emission of the counterparts of the X-ray obscured sources and provide a reliable estimate of their bolometric output.

In this context, Spitzer data have provided a major step forward the understanding of the broad-band properties of the X-ray source populations. If, on one hand, Spitzer data have allowed to pursue the "pioneering" studies of [11] on the spectral energy distributions (SEDs) of broad-line (Type 1), unobscured quasars at higher redshifts (e.g., [12]), on the other hand they have produced significant results in the definition of the SEDs of narrow-line (Type 2), obscured AGN (e.g., [13]).

In this work we aim at providing a robust determination of the bolometric luminosity for hard X-ray selected obscured AGN. This result can be achieved by effectively 
disentangling the nuclear emission related to the active nucleus from the host galaxy starlight, which represents the dominant component (at least for our obscured sources) at optical and near-infrared (near-IR) wavelengths.

\section{SAMPLE SELECTION AND $K_{\mathrm{s}}$-BAND PROPERTIES}

The sources presented in this work were selected from the HELLAS2XMM survey ([3]) which, at the $2-10 \mathrm{keV}$ flux limit of $\approx 10^{-14} \mathrm{erg} \mathrm{cm}^{-2} \mathrm{~s}^{-1}$, covers $\approx 1.4$ square degrees of the sky using XMM-Newton archival pointings ([14]). Approximately $80 \%$ of the HELLAS2XMM sources have a spectroscopic optical classification in the final source catalog (see [15] for details). In particular, we selected eight sources from the original sample of [16] which are characterized by faint (23.7-25.1) $R$-band magnitudes and bright $K_{\mathrm{s}}$-band counterparts $(\approx 17.6-19.1)$; all of our sources are therefore classified as extremely red objects (EROs, $R-K_{\mathrm{S}}>5$ in Vega magnitudes). From the goodquality $K_{\mathrm{s}}$-band images, [16] were able to study the surface brightness profiles of these sources, obtaining a morphological classification. While two sources are associated with point-like objects, the remaining six sources are extended, showing a profile typical of elliptical galaxies. In this latter class of sources, the active nucleus, although evident in the X-ray band, appears hidden or suppressed at optical and near-IR wavelengths, where the observed emission is clearly dominated by the host galaxy starlight. The relatively good constraints on the nuclear emission in the near-IR represent a starting point for the analysis of the Spitzer IRAC and MIPS data.

Due to the faint $R$-band magnitudes of our sources, optical spectroscopy was not feasible even with the 8-m telescope facilities; however, the bright near-IR counterparts of our sources allowed us to obtain spectroscopic redshifts in the $K_{\mathrm{S}}$ band with ISAAC at VLT for two sources: one point-like AGN is classified as a Type 1.9 quasar at a redshift of 2.09, while one extended source has line ratios typical of a LINER at $z=1.35$ (see [17] for further details on these classifications). For the remaining sources, the redshift has been estimated using the optical and near-IR magnitudes, along with the morphological information, as extensively described in $\$ 5.1$ of [16]; all of the redshifts are in the range $\approx 0.9-2.1$. The large column densities $\left[\approx 10^{22}-\mathrm{a}\right.$ few $\left.\times 10^{23} \mathrm{~cm}^{-2}\right]$ and the $2-10 \mathrm{keV}$ luminosities $\left[\approx(1-8) \times 10^{44} \mathrm{erg} \mathrm{s}^{-1}\right.$, once corrected for the absorption] place our sources in the class of the high-luminosity, obscured AGN, the so-called Type 2 quasars (see, e.g., [18] and references therein).

\section{SPITZER DATA}

For our sample of eight sources, we obtained IRAC observations of $480 \mathrm{~s}$ integration time and MIPS observations at $24 \mu \mathrm{m}$ for a total integration time per position of $\approx 1400 \mathrm{~s}$. All of the sources are detected in the four IRAC bands and in MIPS; the faintest source in MIPS has a $24 \mu \mathrm{m}$ flux density of $\approx 150 \mu \mathrm{Jy}$ ( $\approx 5 \sigma$ detection; see [19] for further details on data reduction and cleaning procedures). 


\section{ANALYSIS OF THE TYPE 2 QUASAR SPECTRAL ENERGY DISTRIBUTIONS}

A reliable determination of the bolometric output of our AGN sample requires that the nuclear component, directly related to the accretion processes, is disentangled from the emission of the host galaxy, which provides a dominant contribution in the optical and $K_{\mathrm{S}}$ bands (in the case of extended sources, see [16]). To achieve this goal, we constructed SEDs for all our sources over the optical, near- and mid-IR range. At the same time, we used Spitzer data to improve our previous estimates on the source redshift when possible. In the following, we consider the sample of six extended sources and two point-like objects separately, since a different approach has been adopted for the two sub-samples.

\section{Extended sources}

As already pointed out, from the $K_{\mathrm{s}}$-band morphological analysis carried out by [16], we know that at least up to $2.2 \mu \mathrm{m}$ (observed frame) the stellar contribution is mostly responsible for the emission of these sources. At longer wavelengths, the emission of the active nucleus is expected to arise as reprocessed radiation of the primary emission, while the emission from the galaxy should drop significantly, assuming reasonable elliptical templates. Although many models have been developed in the past to deal with circum-nuclear dust emission (including the effects of the torus geometry and opening angle, grain size distribution and density), in our study we adopted a more phenomenological approach. To reproduce the observed data, we used a combination of two components, one for the host galaxy and another related to the reprocessing of the nuclear emission.

For the galaxy component, we adopted a set of early-type galaxy templates obtained from the synthetic spectra of [20], assuming a simple stellar population spanning a large range of ages (see [19] for details). For the nuclear component, we adopted the templates of [21], which are based on the interpolation of the observed nuclear IR data (at least, up to $\approx 20 \mu \mathrm{m}$ ) of a sample of local AGN through the radiative transfer models of [22]. The strength of such an approach is that the nuclear templates depend upon two quantities, the intrinsic 2-10 keV luminosity (which provides the normalization of the SED) and the column density (responsible for the shape of the SED), and these are known directly from the X-ray spectra ([23]), once the redshift is known.

We also used all the available information, extended over the Spitzer wavelength range, to place better constraints on the source redshift than those reported in [16]. Overall, we find a good agreement with the redshifts presented in [16], although Spitzer allows us to provide estimates with lower uncertainties; only for one source the redshift is significantly lower $(z \approx 1$ instead of $\approx 2)$ and likely more reliable.

The data are well reproduced by the sum of the two components; the emission from the galaxy progressively becomes less important at wavelengths above $\approx 4 \mu \mathrm{m}$ (in the source rest frame), where the nuclear reprocessed emission starts emerging significantly (see Fig. 1, left panel), being dominant in MIPS at $24 \mu \mathrm{m}$. Furthermore, the latter is fully consistent with the upper limits provided in the $K_{\mathrm{s}}$ band by [16]. 

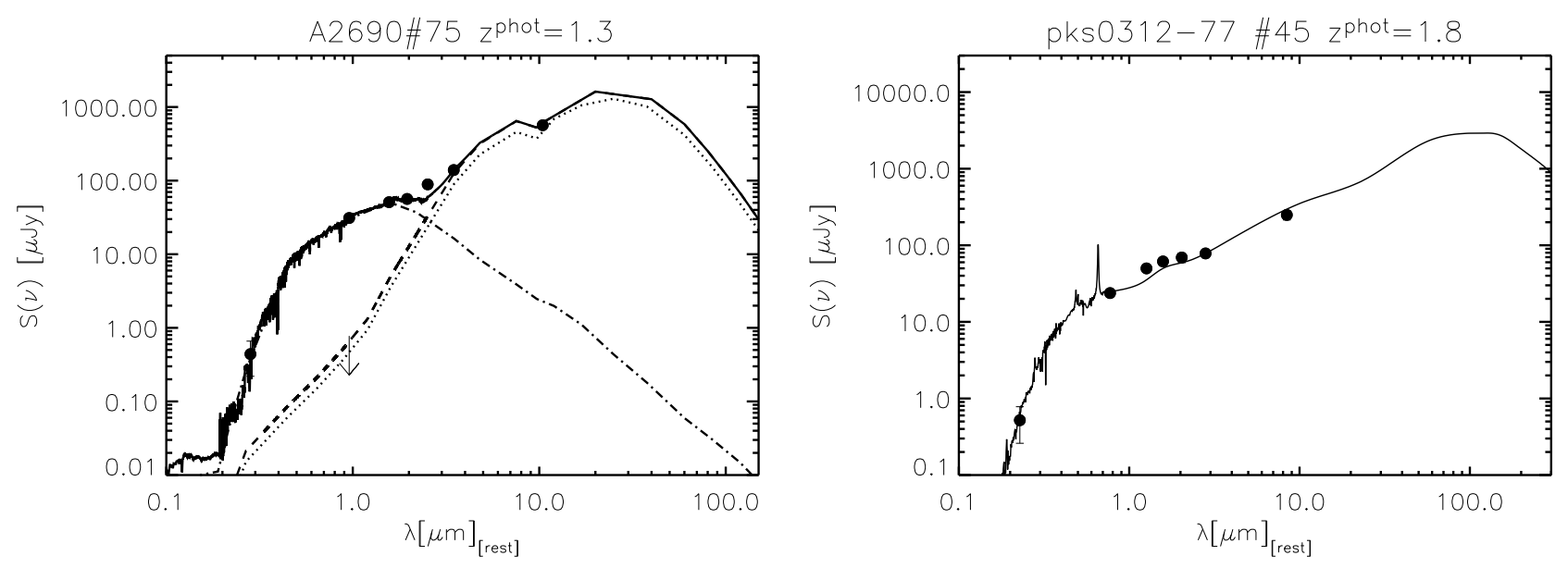

FIGURE 1. Rest-frame SEDs for two representative Type 2 quasars of the current sample: an obscured AGN hosted by an elliptical galaxy (on the left) and a point-like AGN (on the right). (Left) The observed data (filled circles) are reproduced by summing up (solid line) the contribution of an early-type galaxy template (dot-dashed line) to the reprocessed nuclear component (dashed line). The dotted line shows the nuclear component obtained from the templates of [21], normalized using the X-ray luminosity and column density (i.e., without fitting the data; see text and [19] for details). The downward-pointing arrow indicates the constraint on the nuclear emission derived from the $K_{\mathrm{s}}$-band data ([16]). The combination of the two templates is also consistent with the $R$ - $K_{\mathrm{S}}$ color. (Right) The observed data (filled circles) are well reproduced by the red quasar template from [13] (solid line).

\section{Point-like sources}

For the two point-like sources, we adopted a different strategy, since their emission in the near-IR is dominated by the unresolved AGN. To reproduce their observed SEDs, we extincted a Type 1 quasar template from [11] with several extinction laws, but we were not able to find a satisfactory solution. Then we used the recently published red quasar template from [13] and found good agreement with the data (Fig. 11, right panel), consistently with the results obtained for some obscured AGN in the ELAIS-S1 field ([24]). As in the AGN sub-sample described above, most of the uncertainty lies in the far-IR, where a proper study of the SEDs would require MIPS data at 70 and $160 \mu \mathrm{m}$.

\section{BOLOMETRIC CORRECTIONS}

The determination of the SEDs is meant to be the first step toward the estimate of the bolometric luminosities $\left(L_{\text {bol }}\right)$ of obscured AGN. The bolometric luminosities can be estimated from the luminosity in a given band by applying a suitable bolometric correction $k_{\text {bol }}$; typically, to convert the $2-10 \mathrm{keV}$ luminosity into $L_{\mathrm{bol}}, k_{\mathrm{bol}} \approx 30$ is assumed, although this value was derived from the average of few dozens of bright, mostly low-redshift Type 1 quasars ([11]). For obscured sources, only few estimates are present in literature (e.g., [13]). We derived $k_{\text {bol }}$ by integrating the quasar SEDs over the X-ray $(0.5-500 \mathrm{keV})$ and IR $(1-1000 \mu \mathrm{m})$ intervals; in the X-ray band, we converted the $2-10 \mathrm{keV}$ luminosity assuming a power law with photon index $\Gamma=1.9$ (typical for 
AGN emission) and the observed column density ([23]).

To derive the bolometric corrections, we accounted for both the covering factor of the absorbing material (i.e., the opening angle of the torus) and the anisotropy of the IR emission. According to unification models of AGN, the former effect should be directly related to the observed fraction of Type 2/Type 1 AGN which, in the latest models of [6], is $\approx 1.5$ in the luminosity range of our sample. Furthermore, the torus is likely to re-emit a fraction of the intercepted radiation in a direction which does not lie along our line-of-sight; the correction for this anisotropy, according to the templates of [21], is $\approx 10-20 \%$ (given the column densities of our sources). Once these corrections are taken into account, we obtain $\left\langle k_{\mathrm{bol}}\right\rangle \approx 35$ (median $k_{\mathrm{bol}} \approx 26$ ), similar to the average value of [11]; the Type 1.9 quasar at $z=2.09$ has the highest $k_{\text {bol }}(\approx 97)$; see [19] for a discussion on the uncertainties in these estimates.

\section{BLACK HOLE MASSES AND EDDINGTON RATIOS}

For the six AGN hosted by elliptical galaxies, we can derive both the galaxy and black hole masses. Since the near-IR emission is dominated by the galaxy starlight, we computed the rest-frame $L_{\mathrm{K}}$ assuming the appropriate SED templates and then the galaxy masses using $M_{\star} / L_{\mathrm{K}} \approx 0.5-0.9$ ([20]); all of our AGN are hosted by massive galaxies $\left(\approx 1-6 \times 10^{11} \mathrm{M}_{\odot}\right)$.

To estimate the black hole masses, we used the local $M_{\mathrm{BH}}-L_{\mathrm{K}}([25])$ which, along with the $M_{\star} / L_{\mathrm{K}}$ values, provides a $M_{\mathrm{BH}}-M_{\star}$ relation. Despite several attempts in the recent literature to investigate whether and how the black hole mass vs. stellar mass relation evolves with cosmic time, there is no consensus yet. In this work, we assume the findings of [26], who found that in the redshift range covered by our sources, the $M_{\mathrm{BH}}-M_{\star}$ relation evolves by a factor of $\approx 2$ with respect to the local value; see [19] for an extensive discussion. Under this hypothesis, we obtain black hole masses for the six obscured quasars hosted by elliptical galaxies of $\approx 2.0 \times 10^{8}-2.5 \times 10^{9} \mathrm{M}_{\odot}$; these values are broadly consistent with the average black hole masses obtained by [27] for the Sloan Digital Sky Survey (SDSS) Type 1 quasars (using optical and ultra-violet mass scaling relationships) in our redshift range $\left(\approx 3.5 \times 10^{8}-8.6 \times 10^{8} \mathrm{M}_{\odot}\right)$.

As a final step, we derived the Eddington ratios, defined as $L_{\mathrm{bol}} / L_{\mathrm{Edd}}$, where $L_{\mathrm{Edd}}$ is the Eddington luminosity. We note that the uncertainties related to these estimates are clearly large, due to the uncertainties of the approach adopted to derive the bolometric luminosities (through the templates of [21]) and the black hole masses (see above). The average Eddington ratio is $\approx 0.05$, suggesting that our obscured quasars may have already passed their rapidly accreting phase and are reaching their final masses at low Eddington rates. The Eddington ratios of our sources are significantly lower than those derived for the SDSS Type 1 quasars in the same redshift range $(\approx 0.3-0.4$, see [27] $)$.

\section{SUMMARY}

We used optical, near-IR, and Spitzer IRAC and MIPS (at $24 \mu \mathrm{m}$ ) data to unveil the reprocessed nuclear emission of eight hard X-ray selected Type 2 quasars at $z \approx 0.9-2.1$. 
From proper modelling of the nuclear SEDs, we derived a median (average) bolometric correction of $\approx 26(\approx 35)$. For the six obscured sources dominated by the host galaxy starlight up to near-IR wavelengths, we also derived black hole masses of the order of $2.0 \times 10^{8}-2.5 \times 10^{9} \mathrm{M}_{\odot}$ and relatively low Eddington ratios $(\approx 0.05)$, suggestive of a low-activity accretion phase.

\section{ACKNOWLEDGMENTS}

The authors acknowledge partial financial support by the Italian Space Agency under the contract ASI-INAF I/023/05/0.

\section{REFERENCES}

1. R. Giacconi et al., ApJS 139, 369-410 (2002).

2. D. M. Alexander et al., AJ 126, 539-574 (2003).

3. F. Fiore et al., $A \& A$ 409, 79-90 (2003).

4. W. N. Brandt and G. Hasinger, $A R A \& A$ 43, 827-859 (2005).

5. A. Comastri, G. Setti, G. Zamorani and G. Hasinger, $A \& A$ 296, 1-12 (1995).

6. R. Gilli, A. Comastri and G. Hasinger, A\&A 463, 79-96 (2007).

7. F. E. Bauer, D. M. Alexander, W. N. Brandt, D. P. Schneider, E. Treister, A. E. Hornschemeier and G. P. Garmire, AJ 128, 2048-2065 (2004).

8. R. C. Hickox and M. Markevitch, ApJ 645, 95-114 (2006).

9. A. J. Barger, L. L. Cowie, R. F. Mushotzky, Y. Yang, W.-H. Wang, A. T. Steffen and P. Capak, AJ 129, 578-609 (2005).

10. P. F. Hopkins, L. Hernquist, T. J. Cox, T. Di Matteo, B. Robertson and V. Springel, ApJS 163, 1-49 (2006).

11. M. Elvis et al., ApJS 95, 1-68 (1994).

12. G. T. Richards et al., ApJS 166, 470-497 (2006).

13. M. Polletta et al., ApJ 642, 673-693 (2006).

14. A. Baldi, S. Molendi, A. Comastri, F. Fiore, G. Matt and C. Vignali, ApJ 564, 190-195 (2002).

15. F. Cocchia et al., $A \& A$, in press, astro-ph/0612023(2007).

16. M. Mignoli et al., $A \& A$ 418, 827-840 (2004).

17. R. Maiolino et al., $A \& A$ 445, 457-463 (2006).

18. C. Vignali, D. M. Alexander and A. Comastri, MNRAS 373, 321-329 (2006).

19. F. Pozzi et al., A\&A, in press, arXiv:0704.0735(2007).

20. G. Bruzual and S. Charlot, MNRAS 344, 1000-1028 (2003).

21. L. Silva, R. Maiolino and G. L. Granato, MNRAS 355, 973-985 (2004).

22. G. L. Granato and L. Danese, MNRAS 268, 235-252 (1994).

23. G. C. Perola et al., $A \& A$ 421, 491-501 (2004).

24. C. Gruppioni et al., A\&A, in preparation.

25. A. Marconi and L. K. Hunt, ApJ 589, L75-L77 (2003).

26. C. Y. Peng, C. D. Impey, L.C. Ho, E. J. Barton and H.-W. Rix, ApJ 640, 114-125 (2006).

27. R. J. McLure and J. S. Dunlop, MNRAS 352, 1390-1404 (2004). 